\author{
Marquette University \\ e-Publications@Marquette
}

Mechanical Engineering Faculty Research and

Publications

Mechanical Engineering, Department of

2006

\title{
Effect of Keyswitch Design of Desktop and Notebook Keyboards Related to Key Stiffness and Typing Force
}

Marcia J. Bufton

Marquette University

Richard W. Marklin

Marquette University, richard.marklin@marquette.edu

Mark L. Nagurka

Marquette University, mark.nagurka@marquette.edu

Guy G. Simoneau

Marquette University, guy.simoneau@marquette.edu

Follow this and additional works at: https://epublications.marquette.edu/mechengin_fac

Part of the Mechanical Engineering Commons, and the Physical Therapy Commons

\section{Recommended Citation}

Bufton, Marcia J.; Marklin, Richard W.; Nagurka, Mark L.; and Simoneau, Guy G., "Effect of Keyswitch Design of Desktop and Notebook Keyboards Related to Key Stiffness and Typing Force" (2006).

Mechanical Engineering Faculty Research and Publications. 2.

https://epublications.marquette.edu/mechengin_fac/2 
Marquette University

e-Publications@Marquette

\section{Mechanical Engineering Faculty Research and Publications/College of} Engineering

This paper is NOT THE PUBLISHED VERSION; but the author's final, peer-reviewed manuscript. The published version may be accessed by following the link in the citation below.

Ergonomics, Vol. 49, No. 10 (August 2006): 996-1012. DOI. This article is (C) Taylor \& Francis and permission has been granted for this version to appear in e-Publications@Marquette. Taylor \& Francis does not grant permission for this article to be further copied/distributed or hosted elsewhere without the express permission from Taylor \& Francis.

\section{Effect of Keyswitch Design of Desktop and Notebook Keyboards Related to Key Stiffness and Typing Force}

Marcia J. Bufton

Department of Mechanical Engineering, Marquette University, Milwaukee, WI Richard W. Marklin Department of Mechanical Engineering, Marquette University, Milwaukee, WI Mark L. Nagurka Department of Mechanical Engineering, Marquette University, Milwaukee, WI Guy G. Simoneau Department of Physical Therapy, Marquette University, Milwaukee, WI

\section{Abstract}

This study aimed to compare and analyse rubber-dome desktop, spring-column desktop and notebook keyboards in terms of key stiffness and fingertip typing force. The spring-column keyboard resulted in the 
highest mean peak contact force $(0.86 \mathrm{~N})$, followed by the rubber dome desktop $(0.68 \mathrm{~N})$ and the notebook $(0.59 \mathrm{~N})$. All these differences were statistically significant. Likewise, the spring-column keyboard registered the highest fingertip typing force and the notebook keyboard the lowest. A comparison of forces showed the notebook (rubber dome) keyboard had the highest fingertip-to-peak contact force ratio (overstrike force), and the spring-column generated the least excess force (as a ratio of peak contact force). The results of this study could aid in optimizing computer key design that could possibly reduce subject discomfort and fatigue.

\section{Keywords}

Computer keys, Computer keyboard, Typing, Typing force

\section{Introduction}

Although forces required to press computer keyboard keys are low, the repetitive nature of typing may result in enough cumulative fingertip force for those who type extensively throughout the workday to contribute to musculoskeletal disorders (MSDs) of the upper extremity such as carpal tunnel syndrome and tenosynovitis. Rempel and Gerson (1991) published one of the earliest studies concerning the relationship between applied fingertip force and keyboarding. In a comparative study between the amount of force applied by typists to the minimum force required for key activation, they found that typing forces applied by the participants were 2.2 to 4.7 times greater than the minimum force required to depress computer keys as determined from forcedisplacement curves.

Studies published by Armstrong et al. (1994) and Martin et al. (1994) showed similar results between typing forces and keyboard reaction forces. The Armstrong et al. (1994) study focused on determining if users exerted more force on some keyboards than others. Instead of typing on one keyboard, participants used three keyboards with different peak contact force values. The typists exerted 2.5 to 3.9 times more force than the required forces of the three keyboards. The Martin et al. (1994) study found that the participants used three to five times more excess force followed by studies with similar ranges of four to five (Martin et al. 1996) and four to seven (Gerard et al. 1996).

Gerard et al. (1999) furthered keyboard research with a study that incorporated keyswitch stiffness, typing pace, fatigue, subject discomfort, muscle activity and auditory feedback. The keyboards included one spring-column key keyboard with a minimum peak force required to depress a key of $0.72 \mathrm{~N}$ and an audible click upon key activation and three rubber dome keyboards with peak contact forces of $0.28 \mathrm{~N}, 0.56 \mathrm{~N}, 0.83 \mathrm{~N}$ and no audible clicks. They found that participants typed with more force and reported more discomfort while typing on the stiffer keyboard of $0.83 \mathrm{~N}$ peak contact force. As the peak contact force increased, the typing force and electromyographic (EMG) levels for extensor and flexor activity increased for the rubber dome keys. Interestingly, the spring-column keyboard $(0.72 \mathrm{~N}$ peak contact force) had the same ratio between force applied to the keyboard and EMG readings as the $0.28 \mathrm{~N}$ rubber dome board. The authors concluded that the auditory feedback supplied by the click of the spring keys and/or the abrupt force change upon key activation may have reduced overstrike force and resulted in strike force-EMG levels on par with the $0.28 \mathrm{~N}$ keyboard.

These previous studies focused on the relationship of various desktop keyboard and keyswitch designs to key stiffness, fingertip loading, EMG activity of forearm muscles and subject comfort. The keyboards were mostly of the rubber dome key type, although a few utilized the spring-column design. To the authors' knowledge, however, no studies have been published on the biomechanical and subjective assessment of notebook computer keys or on a comparative analysis of notebook and desktop keyboards. Since notebooks are becoming increasingly popular, their effect relative to MSDs is of interest since, for those who use a keyboard extensively each workday, they have the same inherent risk factors of MSDs (high repetition and awkward wrist posture) as conventional desktop PCs. 
The objectives of this present study were:

1. To identify and compare the force-displacement characteristics of notebook (rubber dome key design) and desktop (rubber-dome and spring-column key design) computer keyboards from the same manufacturer under dynamic conditions at normal typing speeds;

2. To compare force-displacement characteristics between various keys within each of the three keyboards (notebook, rubber-dome desktop and spring-column desktop);

3. To evaluate the applied fingertip force (as a function of key resistance and travel distance) during typing on all three keyboards followed by a comparison between keyboards;

4. To compare the peak contact forces and fingertip forces for each keyboard and between keyboards;

5. To examine subjective assessment of ease and comfort for each keyboard and typists' keyboard preference among the three keyboards.

\section{Methods}

To accomplish the specified objectives, the experimental design and analysis of this study were performed in two parts. Part 1 examined the force-displacement characteristics of the keyboards as the keys were depressed and released at different constant velocities. Analysis was performed both within and between keyboards. Part 2 examined the fingertip forces that participants applied to the keys as they typed and provided a comparative analysis between keyboards. In conjunction with this part of the study, qualitative results from subject questionnaires were also analysed and compared. The results from both parts 1 and 2 were then used for a comparison of key stiffness and typing force between keyboards.

\subsection{Experimental design - part 1: an investigation of key stiffness characteristics}

The experimental design was a 3 (keyboard) $\times 4$ (key) $\times 3$ (test speed) factorial design with five replicates of each condition in random order for a total of 180 observations. The three keyboards tested were a desktop rubberdome, a desktop spring-column and the notebook. (To the authors' knowledge, notebook keyboards exclusively use rubber dome key design.) All keyboards were acquired from the same major computer manufacturer and had very minimal prior use.

The next independent variable, key type, involved four keys, the ' $a$ ', ' $e$ ', ' $s$ ' and ' $t$ '. These keys, which account for four of the six most commonly typed keys in the English language (Mayzner and Tresselt 1965), are of similar size and design.

The final independent variable considered was key velocity at three levels: $0.5 \mathrm{~mm} / \mathrm{s} ; 30 \mathrm{~mm} / \mathrm{s}$; and $60 \mathrm{~mm} / \mathrm{s}$. A typing speed of $60 \mathrm{~mm} / \mathrm{s}$ translates to approximately 90 words per min (wpm) (assuming constant speed of depression and release), which is a reasonable typing speed for a trained touch typist (Liu 1999). Likewise, $30 \mathrm{~mm} / \mathrm{s}$ corresponds to approximately $45 \mathrm{wpm}$. The two key velocities offer a realistic range of typing speed, while $0.5 \mathrm{~mm} / \mathrm{s}$ represents a quasi-static condition.

The dependent variable was the peak contact force required to depress the key as measured in N. The 'peak contact travel' (the distance the key travels from rest to peak contact force) was measured in $\mathrm{mm}$.

\subsubsection{Equipment and measurements}

The force-displacement measurements for key stiffness characteristics were acquired through the test system developed by Nagurka et al. (1999). A photograph of the test rig ( figure 1) provides an overall view of its general features and layout. This test rig measured key displacement and key force under controlled velocities of travel. The test rig is described briefly here as it was described in detail in a recent publication (Nagurka and Marklin 2005). The primary feature was the machine-driven probe, which struck the key in either a quasi-static $(0.5 \mathrm{~mm} / \mathrm{s})$ or dynamic mode. The stage-probe assembly was powered by a stepper motor that also controls the 
velocity of the keystroke. At the operator's command, the stage advanced the probe arm at a specified velocity and depressed the key to just above the end range of travel. The stage then reversed direction and returned the probe to its 'home' position at the same velocity the key was depressed. The displacement and force measurements were acquired during probe movement through the use of sensors. An optical encoder, attached to the motorized positioning stage, measured displacement up to $100 \mathrm{~mm}$ of travel with a resolution of $1 \mu \mathrm{m}$. Simultaneous force measurements were acquired through the use of a miniature-sized strain gauge load cell mounted in line with the probe to measure contact force. Once the force signal was acquired, it was then conditioned and amplified for use in a data acquisition system, which converted the signal into force values.

Figure 1. Photograph of test rig used to measure peak contact force in order to depress computer keys.

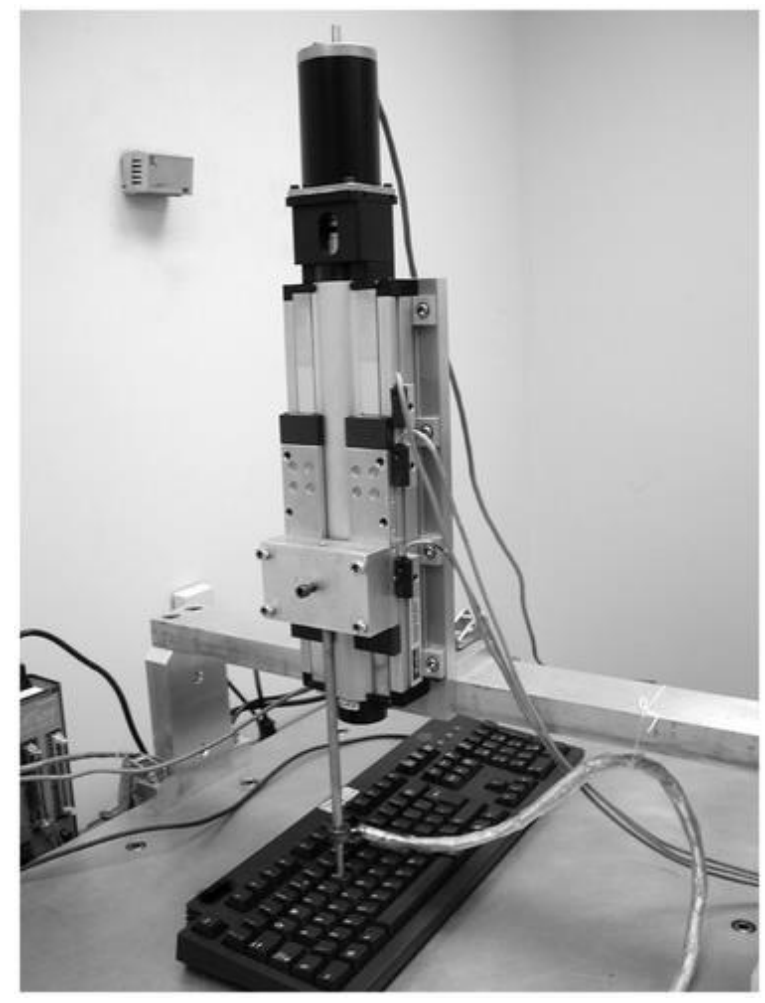

Finally, key velocity was controlled with a range extending from $0.5 \mathrm{~mm} / \mathrm{s}$ to $125 \mathrm{~mm} / \mathrm{s}$. This upper limit represented a typing speed exceeding $100 \mathrm{wpm}$; thus, the test system was more than adequate for measuring forces under typical typing speeds.

\subsubsection{Procedure}

The testing sequence was randomized as follows: (1) the order of type of keyboard was randomized; (2) the order of keys to be tested for each keyboard was randomized; (3) the order of speed for each key was randomized. Five trials were obtained at each test speed for each key for a total of 15 trials per key. When all four keys from a keyboard had been tested in this manner, testing then proceeded to the next ordered keyboard. The key force and displacement data acquired during the keystroke were stored in a data file. The programme then returned to its starting format and a new test velocity was entered for the next data sample.

This procedure was repeated for each of the four selected keys of each of the three keyboards. The test speed was changed for each trial to ensure that trials were not sequentially run at the same speed. Likewise, the order in which keys were tested differed for each keyboard. In this manner, all data samples were acquired by using a set procedure based on a random selection of keys and depression speeds. 


\subsubsection{Statistical methods}

The statistical analysis of key stiffness was designed to observe a spectrum of differences ranging from keys to keyboards. Tests for statistical significance were run to detect differences between keys of a same keyboard and those of different keyboards while also considering the effect of test speed. These factors of test speed and type of key formed the basis for testing the statistical difference between the three keyboards. The statistical results were presented in ANOVA table form using the $p$-value probability approach and were based on the distributive assumption of normality, which is justified due to the large sample size. A detailed comparison between pairs of population means was provided through the use of the Duncan multiple range test.

\subsection{Experimental design - part 2: typing force}

A single factor, full factorial experiment with one independent variable - keyboard - and repeated blocking on participants was used in this part of the study. The dependent variable was the fingertip force transmitted to the keyboard by the subject during typing trials.

\subsubsection{Participants}

Ten female participants were chosen for this study based on various criteria related to health, age, typing speed, skill level and experience. The participants were required to be ten digit ('touch') typists with a minimum typing speed of $40 \mathrm{wpm}$. ('Touch' is defined as the ability to type accurately without looking at the keys; 'ten digit touch typist' is standard nomenclature for trained typists.) Their ages ranged from 19 to 49 years (mean 41.6, SD 8.9) and their typing experience from 5 to 30 years (mean 20.4, SD 7.5). Subject information forms were filled out on all participants including experience with each keyboard type. Additionally, height (mean $164.4 \mathrm{~cm}$, SD 9.3), weight (mean $63.4 \mathrm{~kg}$, SD 11.0) and other anthropometric variables were measured on all participants. Only participants with no previous history of hand paresthesias or related upper extremity pain or disorders within the past 10 years were considered.

\subsubsection{Equipment and measurements}

The testing fixture assembly used to examine typing force was composed of a keyboard fixture with three load cells ( figure 2) and the load cell power supply. The keyboard fixture included two fixture plates, load cells and a wrist support. The three load cells (Sensotec model 31; Sensotec, Columbus, OH, USA) were categorized by their manufacturer as precision miniature load cells of the 'highest accuracy' and calibrated for compression up to $111 \mathrm{~N}$ ( $25 \mathrm{lbs}$ ). They were highly sensitive, with a non-linearity factor of $\pm 0.2 \%$ and a maximum non-repeatability of $\pm 0.05 \%$. Their design was more than adequate for capturing the force signals of this experiment. The load cells were attached in a triangular arrangement beneath the fixture top plate, with two cells on the lower front of the keyboard and one cell on the upper half of the keyboard. The typing forces exerted by the participants were acquired as voltage readings and automatically converted to $\mathrm{N}$. The fixture allowed for interchangability of keyboards through the use of clamps, which securely fastened the keyboards to reduce vibration. A photograph of the test fixture showing the load cell arrangement and wrist support is shown in figure 3.

Figure 2. Side, top and front views of keyboard fixture for measuring force applied to keyboard while participants typed. For more details, see Nagurka and Marklin (2005). 

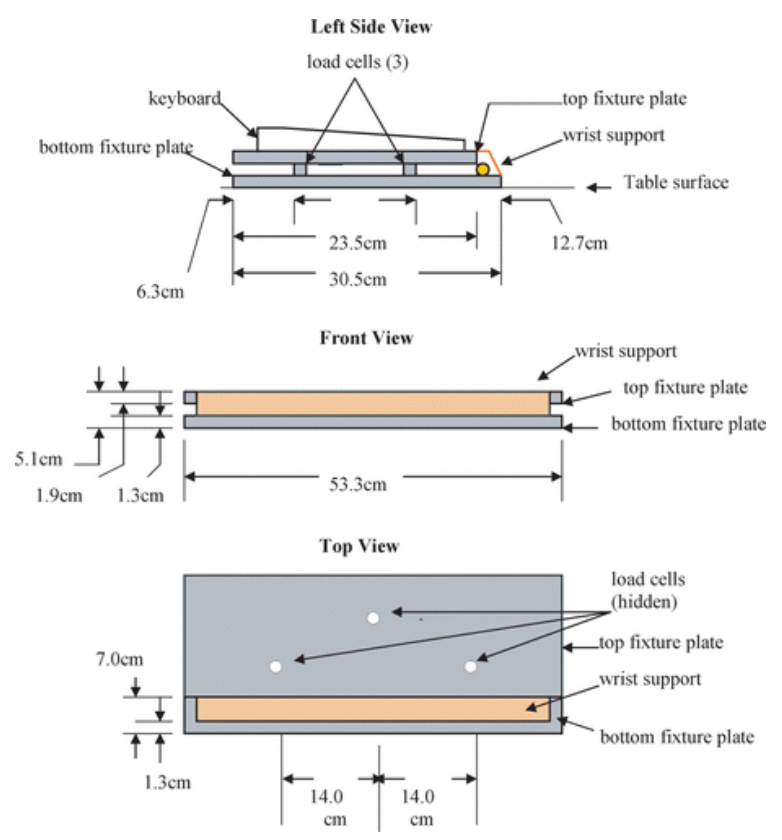

Figure 3. Photograph showing load cell arrangement of test fixture used to measure typing force. The underside of the top plate is exposed, showing the location of the load cells.

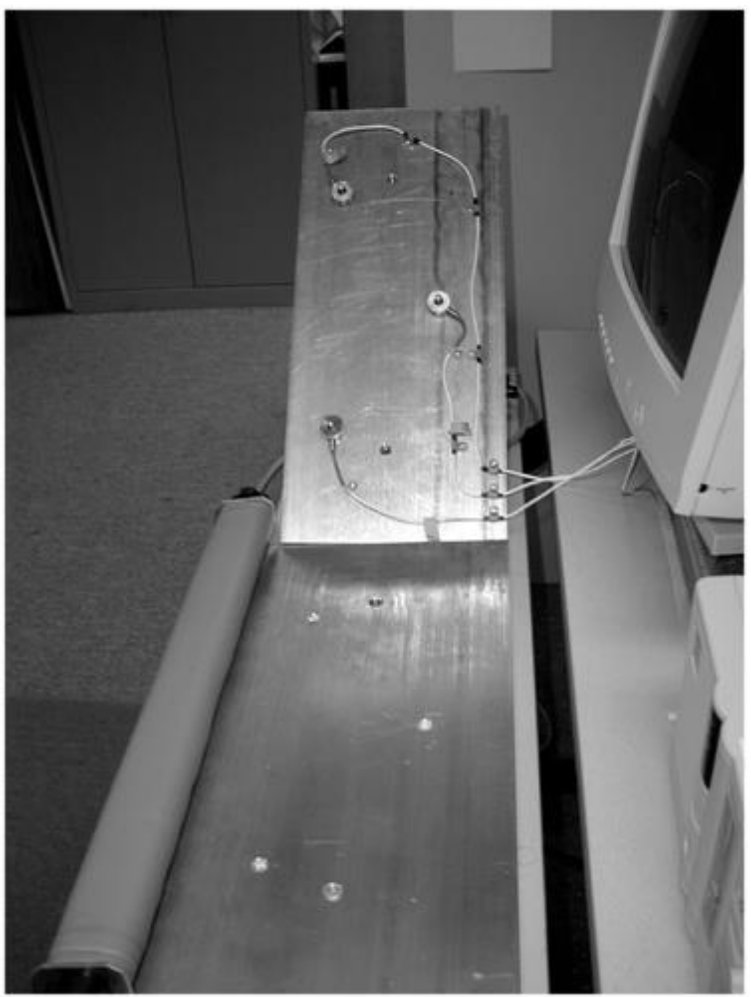

The testing fixture offered several advantages in the measurement of typing force. Keyboards tested on this fixture do not need to be externally modified but are tested 'as is', thereby enhancing the application of the data to actual office settings. In addition, three load cells are used to maximize force acquisition and are recessed into a bottom fixture plate that provides both isolation and protection. A wrist support, which was not attached to the top plate, minimized a force artifact from the weight of hand and wrist bearing on the load cells. Finally, the 
fixture provided the flexibility to accommodate both desktop and notebook keyboard types, which afforded consistency in testing. Figures 4 and 5 illustrate the fixture designs for the desktop and notebook keyboards, respectively.

Figure 4. Photograph of a subject placing her wrist on the wrist support of the keyboard force fixture while typing. The wrist support was attached to the bottom plate and forces applied to the wrist support were not recorded by the load cells.

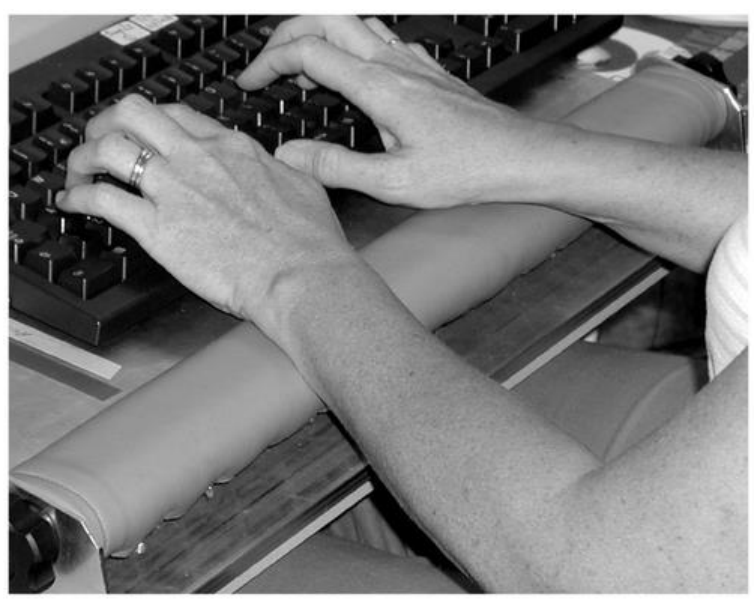

Figure 5. Keyboard force fixture with notebook computer and wrist support.

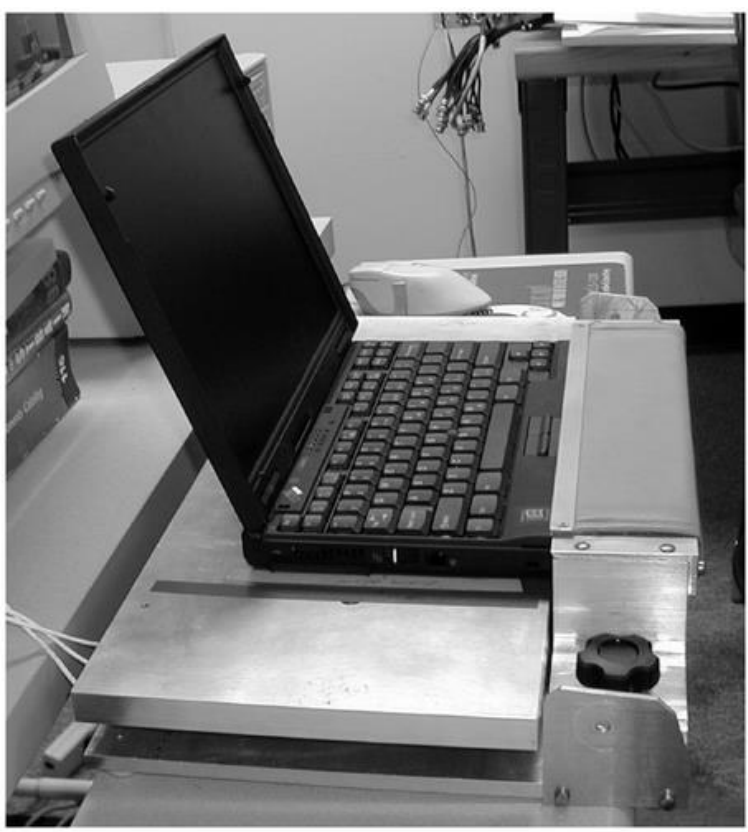

\subsubsection{Procedure}

1. Measurement of anthropometric dimensions of participants.

2. Adjustment of work station according to American National Standards Institute/Human Factors and Ergonomics Society (1988) guidelines.

3. Subject testing using all three keyboards (rubber-dome desktop, spring-column desktop, notebook) from part 1 of this study (keyboard order determined through Latin square designs).

4. A 3-min practice period for acclimatization. 
5. A 6-min typing session for the acquisition of five data trials - each of $30 \mathrm{~s}$ duration - attained in random time intervals throughout the 6-min session.

6. A 5-min rest period between keyboard testing, during which participants completed a questionnaire rating the tactile feel of the keyboard.

Participants were further instructed to type at their preferred speeds based on previous studies that found no relationship between typing speed and fingertip peak force when individuals typed at their preferred speeds (Sommerich et al. 1996, Feuerstein et al. 1997) and that participants activated fewer muscles than those typists working under stressful conditions (Lundervold 1958). In addition, Gerard et al. (2002) found that typists forced to test their maximum typing speeds may suffer significant short-term discomfort.

The above procedures were repeated until all three keyboards had been tested. After the last keyboard had been tested, the participants were asked to complete a keyboard preference form (Likert type scale), in which they ranked keyboards based on preference.

\subsubsection{Statistical methods}

The ANOVA method of statistical analysis based on a block design was used to study significant differences between keyboards relative to typing forces. As in part 1, the data results were analysed using the probability of $p$-values. The number of observations, 30, was large enough for an assumption of normal distribution. From test data of all trials pooled, basic statistics were also determined, such as the mean, the 50th percentile, the 90th percentile, and the 95th percentile.

\section{Results}

\subsection{Part 1: key stiffness - comparison of peak contact force between keyboards}

Typical of the many plots obtained for various keys and speeds is that shown in figure 6 . Note the location of the 'peak contact force' (also known as 'peak force') and peak travel (peak displacement). This particular plot illustrates the general force-displacement relationship between the three test keyboards and, in particular, shows a keyboard comparison of peak contact force for the ' $\mathrm{s}$ ' key at a realistic typing speed of $60 \mathrm{~mm} / \mathrm{s}$.

Figure 6. Force-displacement plot from depressing the ' $\mathrm{s}$ ' key at $60 \mathrm{~mm} / \mathrm{s}$ for all test keyboards. $\mathrm{X}$ represents the peak contact force for each keyboard. Peak travel is the displacement corresponding to the peak contact force.

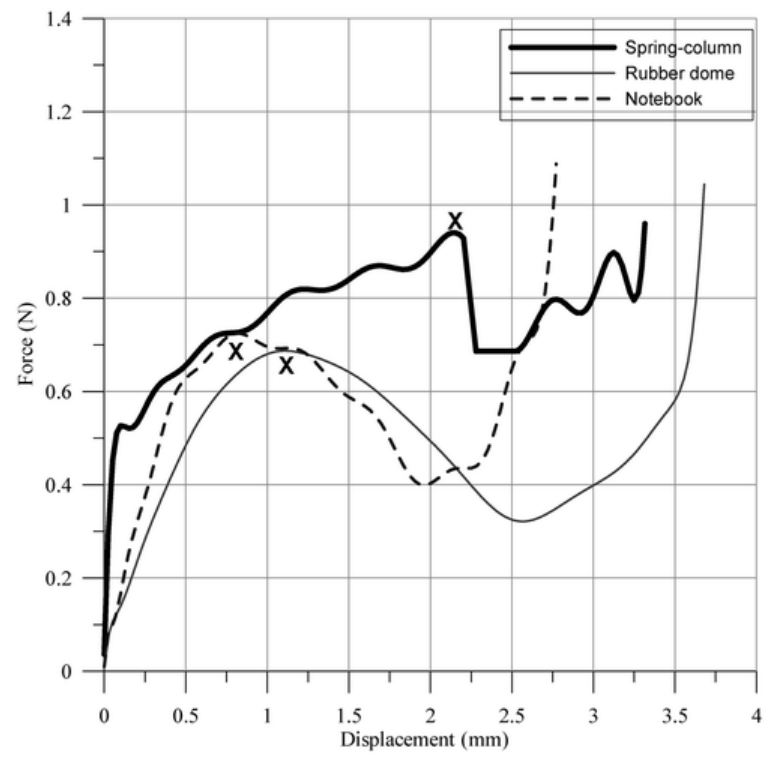


When keyboards were compared over all keys and speeds, the spring-column resulted in the highest peak contact force, followed by the rubber-dome desktop; the notebook displayed the lowest peak contact force ( table 1). Upon further analysis, this comparative keyboard ranking also held true for all three test speeds. At a speed of $0.5 \mathrm{~mm} / \mathrm{s}$, the spring-column keyboard showed the highest average peak contact force across all keys and the notebook the lowest (table 2). This trend was also observed at test speeds of $30 \mathrm{~mm} / \mathrm{s}$ and $60 \mathrm{~mm} / \mathrm{s}$ (tables 3 and 4).

Table 1. Summary of mean (SD) peak contact force for each keyboard $(n=60)$.

\begin{tabular}{|l|l|c|}
\hline & Means of peak contact force (N) & \\
\hline Rubber-dome & Spring-column & Notebook \\
\hline $0.68(0.028)$ & $0.86(0.065)$ & $0.59(0.072)$ \\
\hline
\end{tabular}

Table 2. Mean (SD) of peak contact forces when the keys were depressed and released at $0.5 \mathrm{~mm} / \mathrm{s}$ constant velocity.

\begin{tabular}{|l|l|l|l|l|}
\hline Key & $\begin{array}{l}\text { Rubber-dome } \\
\text { desktop }(\mathbf{n}=\mathbf{5})\end{array}$ & $\begin{array}{l}\text { Spring-column } \\
\text { desktop }(\mathbf{n}=\mathbf{5})\end{array}$ & $\begin{array}{l}\text { Notebook } \\
\mathbf{( n = 5 )}\end{array}$ & $\begin{array}{l}\text { Average (SD) across } \\
\text { all keyboards }(\mathbf{n}=\mathbf{1 5})\end{array}$ \\
\hline $\mathrm{a}$ & $0.71(0.008)$ & $0.77(0.005)$ & $0.49(0.007)$ & $0.66(0.123)$ \\
\hline $\mathrm{e}$ & $0.64(0.009)$ & $0.76(0.008)$ & $0.54(0.005)$ & $0.65(0.091)$ \\
\hline $\mathrm{s}$ & $0.68(0.008)$ & $0.82(0.005)$ & $0.65(0.008)$ & $0.72(0.075)$ \\
\hline $\mathrm{t}$ & $0.63(0.004)$ & $0.84(0.009)$ & $0.52(0.002)$ & $0.67(0.137)$ \\
\hline $\begin{array}{l}\text { Average (SD) across all keys } \\
(\mathrm{n}=20)\end{array}$ & $0.66(0.034)$ & $0.80(0.035)$ & $0.55(0.062)$ & \\
\hline
\end{tabular}

The means of peak contact force of each key were significantly different across all keyboards and for all keyboard pairs at $p<0.05$ level.

Table 3. Mean (SD) of peak contact forces when the keys were depressed and released at $30 \mathrm{~mm} / \mathrm{s}$ constant velocity.

\begin{tabular}{|l|c|c|c|c|}
\hline \multicolumn{1}{|c|}{ Key } & $\begin{array}{c}\text { Rubber-dome } \\
\text { desktop }(\mathbf{n}=\mathbf{5})\end{array}$ & $\begin{array}{c}\text { Spring-column } \\
\text { desktop }(\mathbf{n}=5)\end{array}$ & $\begin{array}{c}\text { Notebook } \\
(\mathbf{n}=5)\end{array}$ & $\begin{array}{c}\text { Average across all } \\
\text { keyboards }(\mathbf{n}=15)\end{array}$ \\
\hline $\mathrm{a}$ & $0.71(0.006)$ & $0.83(0.012)$ & $0.53(0.007)$ & $0.69(0.125)$ \\
\hline $\mathrm{e}$ & $0.66(0.005)$ & $0.83(0.005)$ & $0.59(0.005)$ & $0.69(0.104)$ \\
\hline $\mathrm{s}$ & $0.68(0.004)$ & $0.92(0.009)$ & $0.71(0.005)$ & $0.77(0.110)$ \\
\hline $\mathrm{t}$ & $0.66(0.005)$ & $0.93(0.007)$ & $0.57(0.007)$ & $0.72(0.157)$ \\
\hline Average (SD) across all keys $(\mathrm{n}=20)$ & $0.68(0.019)$ & $0.88(0.049)$ & $0.60(0.067)$ & \\
\hline
\end{tabular}

The means of peak contact force of each key were significantly different across all keyboards and for all keyboard pairs at $p<0.05$ level.

Table 4. Mean (SD) of peak contact forces when the keys were depressed and released at $60 \mathrm{~mm} / \mathrm{s}$ constant velocity.

\begin{tabular}{|l|l|l|l|l|}
\hline Key & $\begin{array}{l}\text { Rubber-dome } \\
\text { desktop }(\mathbf{n}=\mathbf{5})\end{array}$ & $\begin{array}{l}\text { Spring-column } \\
\text { desktop }(\mathbf{n}=\mathbf{5})\end{array}$ & $\begin{array}{l}\text { Notebook } \\
\mathbf{( n = 5 )}\end{array}$ & $\begin{array}{l}\text { Average across all } \\
\text { keyboards }(\mathbf{n}=\mathbf{1 5})\end{array}$ \\
\hline $\mathrm{a}$ & $0.72(0.007)$ & $0.86(0.011)$ & $0.55(0.009)$ & $0.71(0.131)$ \\
\hline $\mathrm{e}$ & $0.66(0.008)$ & $0.86(0.008)$ & $0.60(0.003)$ & $0.71(0.111)$ \\
\hline $\mathrm{s}$ & $0.69(0.007)$ & $0.94(0.005)$ & $0.72(0.004)$ & $0.78(0.115)$ \\
\hline $\mathrm{t}$ & $0.68(0.011)$ & $0.96(0.010)$ & $0.58(0.005)$ & $0.74(0.165)$ \\
\hline
\end{tabular}




\begin{tabular}{|l|l|l|l|}
\hline Average across all keys $(n=20)$ & $0.69(0.024)$ & $0.90(0.047)$ & $0.61(0.066)$ \\
\hline
\end{tabular}

The means of peak contact force for each key were significantly different across all keyboards and for all keyboard pairs at $p<0.05$ level.

The same keyboard order was observed in the magnitude of key travel required for peak contact force. The spring-column keyboard showed the greatest magnitude of peak key travel, ranging from $1.75 \mathrm{~mm}$ to $2.50 \mathrm{~mm}$ depending on speed. The rubber-dome desktop keyboard had the next greatest peak key travel of approximately $1.10 \mathrm{~mm}$ and the notebook keyboard had the smallest magnitude of travel, $0.75 \mathrm{~mm}$.

It was further observed that the mean peak contact force monotonically increased with increased test speed across the test keyboards as shown in figure 7. Both ANOVA and Duncan test analyses showed significant differences in peak contact force between all test speeds across all keyboards (figure 8) when the data from all four keys ('a', 'e', 's', 't') were pooled and analysed as a group.

Figure 7. Mean peak contact force of test keyboards plotted according to speed of depression and release. Each point is the mean force recorded for all force keys $(a, e, s, t) . n=20$ trials for each point.

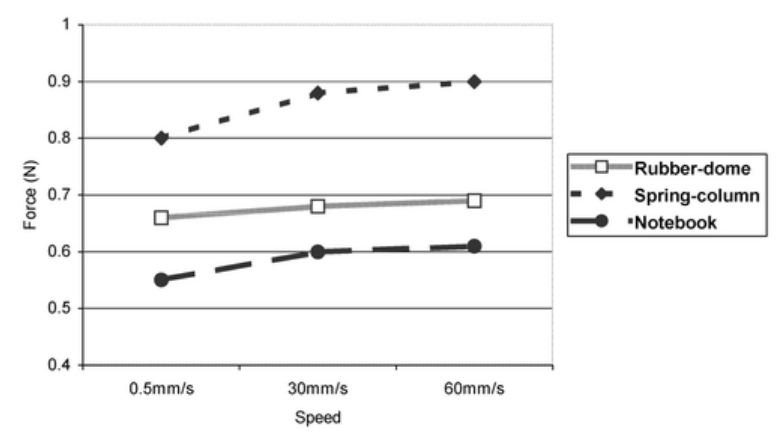

Figure 8. Speed effect on mean peak force when force data were pooled and analysed. The percentage values express the increase in peak contact force compared to the $0.5 \mathrm{~mm} / \mathrm{s}$ test speed.

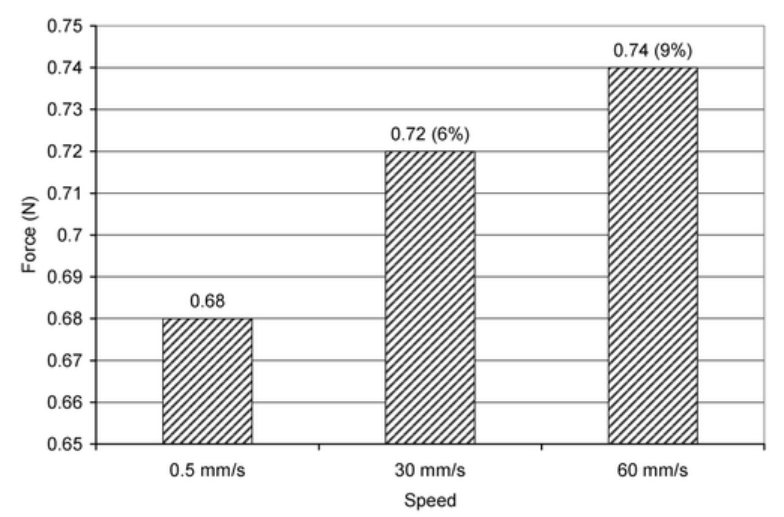

\subsection{Comparison of peak contact force between keys within keyboards at various test speeds}

To provide a comparative study of keys within an individual keyboard, a one-way ANOVA analysis was run for all test speeds and all four keys for each keyboard. In general, all ANOVA tests showed significant differences between the four test keys for each keyboard at each speed. Key forces over all three test speeds ranged from $0.63 \mathrm{~N}$ to $0.72 \mathrm{~N}$ for the rubber-dome desktop keyboard and $0.76 \mathrm{~N}$ to $0.96 \mathrm{~N}$ for the spring-column keyboard. The notebook keyboard showed the widest range of key force, $0.49 \mathrm{~N}$ to $0.72 \mathrm{~N}$. Specifically, post hoc tests of the desktop rubber dome and spring-column keyboards found some key pairs with no significant differences at 
certain test speeds. However, all key pairs within the notebook keyboard were found to be significantly different regardless of speed.

There was no consistent pattern for keys across keyboards relative to peak contact force. For each test speed, key ' $a$ ' generated the greatest peak contact force for the rubber-dome keyboard (0.71 to $0.72 \mathrm{~N})$, key ' $t$ ' for the spring-column keyboard ( 0.84 to $0.96 \mathrm{~N})$, and key ' $s$ ' for the notebook keyboard ( 0.65 to $0.72 \mathrm{~N})$. However, the remaining keys of the desktop keyboards showed no consistent order of peak force for varying test speeds. Alternatively, the notebook keys were consistent in order of peak force and followed a set trend of ' $s$ ', ' $e$ ', ' $t$ ' and 'a' (with 's' exhibiting the greatest force and 'a' the least) for each test speed.

\subsection{Part 2: fingertip typing force}

The summary statistics of typing force applied by participants to keyboards consisted of four areas of interest: mean fingertip typing force, and 50th, 90th and 95th percentiles. Table 5 provides a statistical comparison that suggests similarities in means between the rubber-dome and spring-column desktop keyboards, but not between the desktop and notebook keyboards. These similarities and differences are depicted in figure 9, which shows the comparatively similar and higher typing forces of the desktop keyboards (rubber-dome and springcolumn) as opposed to those of the notebook keyboard.

Table 5. A comparison of peak contact force (part 1) and fingertip force (part 2) for all test keyboards.

\begin{tabular}{|l|l|l|l|}
\hline & Rubber-dome desktop & Spring-column desktop & Notebook \\
\hline Mean (SD) peak contact force (Part 1) & $0.68 \mathrm{~N}(0.028)$ & $0.86 \mathrm{~N}(0.065)$ & $0.59 \mathrm{~N}(0.072)$ \\
\hline Mean fingertip force (Part 2) & $1.54 \mathrm{~N}(0.92)$ & $1.55 \mathrm{~N}(0.99)$ & $1.41 \mathrm{~N}(0.82)$ \\
\hline Fingertip force at 50th percentile & $1.34 \mathrm{~N}$ & $1.35 \mathrm{~N}$ & $1.22 \mathrm{~N}$ \\
\hline Fingertip force at 90th percentile & $2.80 \mathrm{~N}$ & $2.88 \mathrm{~N}$ & $2.54 \mathrm{~N}$ \\
\hline Fingertip force at 95th percentile & $3.27 \mathrm{~N}$ & $3.42 \mathrm{~N}$ & $2.98 \mathrm{~N}$ \\
\hline
\end{tabular}

No statistically significant differences in fingertip force were found between the desktop keyboards (rubberdome and spring-loaded) for mean or percentiles. All differences in mean and percentiles of fingertip force between the notebook and the two desktop keyboards were statistically significant at $p<0.05 . \mathrm{N}=60$ for peak contact forces; $n=50$ for fingertip forces.

Figure 9. Mean and percentiles of fingertip typing force for each keyboard. Each point is the mean force recorded for $n=50$ trials.

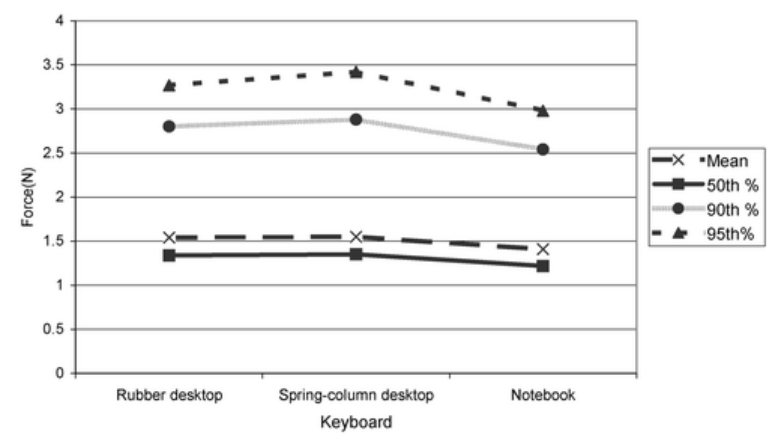

An ANOVA analysis of the force percentiles statistically determined that all three keyboards were significantly different. However, post hoc testing of keyboard pairs at each percentile discerned no significant difference between the spring and rubber-dome desktop keyboards, but a significant difference when each was paired with the notebook keyboard. 


\subsection{Qualitative survey results}

Each subject completed two survey forms, the Keyboard Assessment form (visual analogue scale) and the Keyboard Preference form (Likert scale). The Keyboard Assessment form was completed for each keyboard and consisted of six questions relevant to comfort and feedback. The Keyboard Preference form required only that the subject rank the keyboards in terms of preference.

A one-way ANOVA analysis was performed on each question to determine which questions resulted in significantly different responses between keyboards. Questions concerning key stroke, tactile feedback, typing ease and keyboard comfort were statistically determined to not be significantly different between all three keyboards. However, questions relevant to key feel and audio feedback showed significant differences in their responses between all test keyboards. According to survey responses on key feel, the spring keys were judged too stiff, the notebook keys too soft and the rubber-dome keys closest to optimum value. On the question of audio feedback, the spring-column and notebook keyboards were considered to have the highest and lowest audio feedbacks, respectively.

The participants were also asked to complete a survey form on keyboard preference with three options: (1) most preferred; (2) neutral; (3) least preferred. The spring-column keyboard secured the greatest number of most preferred and least preferred responses. The notebook was a neutral choice for most participants, while the rubber-dome desktop responses appeared somewhat evenly divided between all three categories.

\subsection{Comparison of peak contact force and applied fingertip force}

The relationship between the peak contact force required for character generation and the amount of fingertip force applied for each test keyboard was of considerable interest in this study, as shown in table 6 . The springcolumn keyboard had the highest peak contact force and the notebook keyboard had the lowest; yet, participants applied the greatest percentage of excess typing force to the notebook keyboard and the least to the spring-column keyboard. This was true for all mean and percentile levels, as shown in figure 10.

Table 6. A ratio comparison of fingertip force $(\mathrm{N})$ to peak contact force $(\mathrm{N})$ for all test keyboards.

\begin{tabular}{|l|l|l|l|}
\hline & $\begin{array}{l}\text { Rubber- } \\
\text { dome }\end{array}$ & $\begin{array}{l}\text { Spring- } \\
\text { column }\end{array}$ & Notebook \\
\hline Ratio of mean fingertip force $(\mathrm{N})$ to peak contact force $(\mathrm{N})$ & 2.2 & 1.8 & 2.4 \\
\hline Ratio of fingertip force at 50th percentile (N) to peak contact force (N) & 1.9 & 1.5 & 2.0 \\
\hline Ratio of fingertip force at 90th percentile (N) to peak contact force (N) & 4.1 & 3.3 & 4.3 \\
\hline Ratio of fingertip force at 95th percentile (N) to peak contact force (N) & 4.8 & 3.9 & 5.0 \\
\hline
\end{tabular}

Figure 10. Ratio of mean and percentiles of fingertip typing force $(N)$ to peak contact force $(N)$ for each keyboard in fixture testing (part 1). Each point is the mean of $n=60$ trials for peak contact force and $n=50$ trials for fingertip typing force.

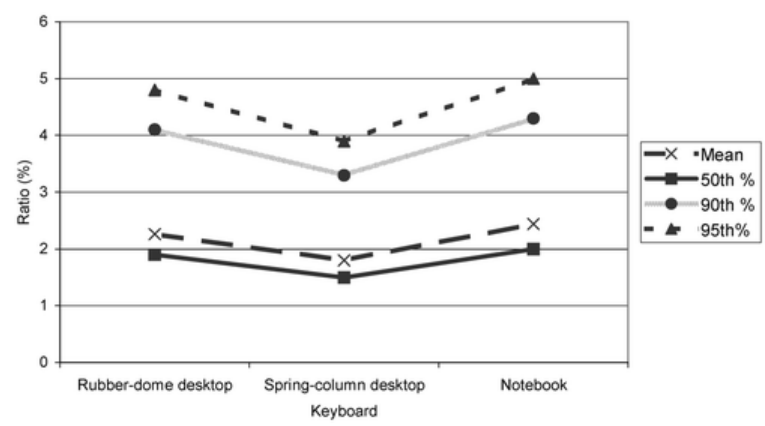




\section{Discussion}

The spring-column keyboard exhibited the highest peak contact force and fingertip force, and the notebook displayed the lowest peak and fingertip forces for all percentiles. However, a ratio comparison of peak and fingertip forces for each keyboard found the notebook keyboard as having the highest fingertip-to-peak contact force ratio for each percentile. Accordingly, the participants used more excess force (also referred to as overstrike force) on the notebook keyboard than on the other two keyboards, even though the notebook had the lowest key stiffness and fingertip typing force. However, the spring-column keyboard, which registered the highest peak contact forces, displayed the lowest force ratios. All of the above overstrike force ratios from this study (1.8 to 5.0) compare well to those of previous studies: 2.2 to 4.7 (Rempel and Gerson 1991), 2.5 to 3.9 (Armstrong et al. 1994), 3 to 5 (Martin et al. 1994), 4 to 5 (Martin et al. 1996) and 4 to 7 (Gerard et al. 1996).

The Gerard et al. (1999) study looked at both rubber-dome and spring desktop keyboards. They found that participants used less overstrike force on spring-column keyboards than on rubber-dome keyboards of less peak contact force, which is consistent with findings from this study. The authors surmised that the auditory feedback supplied by the click of the spring keys and/or the abrupt force change after reaching peak travel may have reduced the overstrike force. This appears to be a reasonable hypothesis, particularly when considering that participants assessed the spring-column keyboard highest in auditory feedback. Possible factors causing excessive overstrike force for the notebook keyboards may be that the notebook keys exhibited the shortest peak travel and overall travel distance (see figure 6) and lowest key stiffness of the test keyboards (0.59N). Participants assessed the notebook key stiffness on the 'too soft' end of the subjective scale. The soft key feel may hinder tactile feedback that signals when the peak contact force has been reached, resulting in excess typing force.

Another trend observed among all three keyboards was that peak contact force increased with increasing test speed. This increase was as high as $9 \%$ for $60 \mathrm{~mm} / \mathrm{s}$ compared to $0.5 \mathrm{~mm} / \mathrm{s}$ (see figure 8). This relationship between contact force and displacement rate is indicative of damping (viscous-type) and was discussed by Nagurka and Marklin (2005). They observed that if the key mechanism follows a viscous damping model, then force will increase with increasing depression speed since damping dissipates the energy imparted to the key from the fingertip. Therefore, at speeds greater than measured in this study $(60 \mathrm{~mm} / \mathrm{s})$, damping may play a more dominant role in total contact force. Under conditions of viscous damping, Nagurka and Marklin suggest that there may be a range of damping that minimizes contact force yet optimizes tactile feedback.

The peak contact force generated by the four test keys on an individual keyboard were significantly different from each other, notwithstanding the fact that all four test keys used the same keycap size and key mechanism within each keyboard. This observation was valid for all three keyboards and indicates variance in the manufacturing processes. Although the differences among keys was statistically significant in this study, these differences may not be noticeable to the user according to the Just Noticeable Difference (JND) theory (Ikei et al. 1997). In this research study, the rubber-dome desktop keyboard showed the lowest percentage of difference between keys with an average value of $8.2 \%$ while the notebook displayed the highest difference - a $32 \%$ difference between keys. A user would perhaps be more inclined to notice a difference in peak contact force between keys of a notebook keyboard than with a rubber-dome desktop keyboard, if such differences achieved a JND level.

In terms of keyboard preference, participants either emphatically liked or disliked the spring-column keyboard, while they were more neutral about the notebook and rubber-dome desktop keyboards. This finding may be due to the fact that spring-column keyboards are rarely used in office settings now and, to the authors' knowledge, are not shipped with most new PCs to customers. Thus, participants in this study may not have liked spring-column keyboards because of their unfamiliarity with them. 
Both keyboard and fingertip testing were performed using testing fixtures with distinct advantages. The keyboard test rig ( figure 1) can quantify force-displacement (under constant velocity) at both pseudo-static and typical typing speeds to obtain damping. The fingertip test fixture (figure 4) accommodates keyboards without any external modification and also provides a wrist support to minimize a force artefact from hand, wrist or forearm weight. Both testing fixtures provide easy keyboard set-up and the flexibility to accommodate both desktop and notebook keyboard types.

\section{Conclusions}

- Over all test keys and speeds, the spring-column keyboard recorded the highest mean peak contact force, $0.86 \mathrm{~N}$, followed by the rubber-dome keyboard with $0.68 \mathrm{~N}$ and the notebook keyboard with 0.59 N.

- Across all three keyboards, the mean peak contact force increased monotonically as depression speed increased.

- During typing sessions, participants applied the least fingertip force to the notebook keyboard keys, but also applied the most excess force to notebook keys; by contrast, participants applied the least overstrike force to the spring-column keys.

- There were significant differences in peak contact force between test keys of the same key design and mechanism within each keyboard, regardless of depression speed.

- The spring-column keyboard was either liked or disliked while the rubber-dome desktop and notebook received more moderate ratings.

- The force overstrike observed in the notebook fingertip-force tests might be reduced by increased tactile feedback and travel distance in key design.

\section{Acknowledgement}

The authors express their gratitude to the Office Ergonomics Research Committee (OERD) for providing the funds to build the test rig that measured peak contactforces.

\section{References}

American National Standards Institute/Human Factors and Ergonomics Society. 1988. "American national standard for human factors engineering of visual display terminal workstation, 100-1988". Available online at: http://www.hfes.org/Publications/ANSI-HFS-100.html

Armstrong, T., Foulke, J., Martin, B., Gerson, J. and Rempel, D. 1994. Investigation of applied force in alphanumeric keyboard work. Journal of the American Industrial Hygiene Association, 55: 30-35.

Feuerstein, M., Armstrong, T., Hickey, P. and Lincoln, A. 1997. Computer keyboard force and upper extremity symptoms. Journal of Occupational and Environmental Medicine, 39: 1144-1153.

Gerard, M., Armstrong, T., Foulke, J. and Martin, B. 1996. Effects of key stiffness on force and development of fatigue while typing. American Industrial Hygiene Association Journal, 57: 849-854.

Gerard, M., Armstrong, T., Franzblau, A., Martin, B. and Rempel, D. 1999. The effects of keyswitch stiffness on typing force, finger electromyography, and subjective discomfort. American Industrial Hygiene Association Journal, 60: 762-769.

Gerard, M., Armstrong, T. and Martin, B. 2002. Short and long term effects of auditory feedback on typing force, EMG and comfort while typing. Applied Ergonomics, 33: 129-138.

Ikei, Y., Wakamatsu, K. and Fukuda, S. 1997. Vibratory tactile display of image-based texture. IEEE Computer Graphics and Applications, 17: 53-61.

Liu, K. 1999. "Design of a test rig for measurement of stiffness and damping characteristics of computer keyboard keys". Milwaukee, WI: Marquette Univerisity. Master's thesis 
Lundervold, A. 1958. Electromyographic investigations during typing. Ergonomics, 1: 226-233.

Martin, B., Armstrong, T., Foulke, J., Natarajan, S., Klinenberg, E., Serina, E. and Rempel, D. 1994. Finger forces during computer keyboard work part I: relation of keyboard reaction force to finger flexor muscles surface EMG. Occupational Health and Safety, 2: 198-200.

Martin, B., Armstrong, T., Fouke, J., Natarajan, S., Klinenberg, E., Serina, E. and Rempel, D. 1996. Keyboard reaction force and finger flexor electromyograms during computer keyboard work. Human Factors, 38: 654-664.

Mayzner, M. S. and Tresselt, M. E. 1965. Tables of single-letter and diagram frequency counts for various wordlength and letter-position combinations. Psychonomic Monograph Supplements, 1: 13-33.

Nagurka, M. and Marklin, R. 2005. Measurement of stiffness and damping characteristics of computer keyboard keys. Journal of Dynamic Systems, Measurement, and Control, 127: 283-288.

Nagurka, M., Marklin, R. and Liu, K. 1999. Design of a test rig for measurement of stiffness and damping characteristics of computer keyboard keys. Proceedings of the 1999 American Control Conference. June2-41999, San Diego, CA. pp.1749-1753.

Rempel, D. and Gerson, J. 1991. Fingertip forces while using three different keyboards. Proceedings of the Human Factors Society 35th Annual Meeting. September2-61991, San Francisco, CA. pp.253-255.

Sommerich, C., Marras, W. and Parnianpour, M. 1996. A quantitative description of typing biomechanics. Journal of Occupational Rehabilitation, 6: 33-55. 